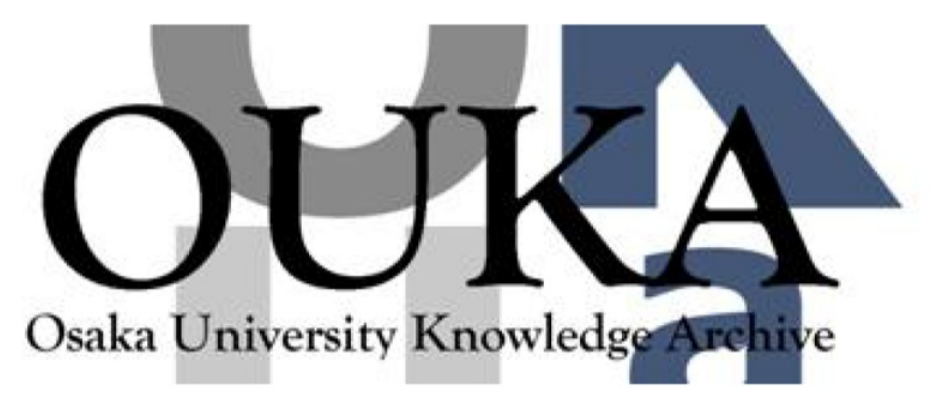

\begin{tabular}{|c|l|}
\hline Title & $\begin{array}{l}\text { Realization of number-phase minimum-uncertainty } \\
\text { states and number states by quantum } \\
\text { nondemolition measurement }\end{array}$ \\
\hline Author(s) & Kitagawa, M.; Imoto, N.; Yamamoto, Y. \\
\hline Citation & Physical Review A. 35(12) p.5270-p. 5273 \\
\hline Issue Date & $1987-06-01$ \\
\hline oaire:version VoR \\
\hline URL & https://hdl. handle. net/11094/77659 \\
\hline rights & $\begin{array}{l}\text { Copyright (1987) by the American Physical } \\
\text { Society }\end{array}$ \\
\hline Note & \\
\hline
\end{tabular}

Osaka University Knowledge Archive : OUKA

https://ir. Library. osaka-u. ac. jp/

0saka University 


\title{
Realization of number-phase minimum-uncertainty states and number states by quantum nondemolition measurement
}

\author{
M. Kitagawa, N. Imoto, and Y. Yamamoto \\ NTT Electrical Communications Laboratories, 3-9-11 Midori-cho, Musashino-shi, Tokyo 180, Japan
}

(Received 24 November 1986)

\begin{abstract}
Number-phase minimum uncertainty states and number states are shown to be realized by quantum nondemolition measurements of the photon number. Interphase modulation in a Kerr medium establishes a quantum-mechanical correlation between the signal and probe waves initially in coherent states. State reduction is realized by homodyne detection of a probe quadrature-phase amplitude. The number uncertainty can be arbitrarily reduced by increasing the probe intensity. The minimum uncertainty relation between the number and sine operators approximately holds until a near-number state is realized.
\end{abstract}

A number-phase minimum uncertainty state (NUS) is mathematically defined as an eigenstate of the operator $e^{r} \hat{n}+i e^{-r} \hat{S}, 1$ where $\hat{n}$ is the number operator, $\hat{S}$ $=(\hat{n}+1)^{-1 / 2} \hat{a} / 2 i+$ H.c. (the sine operator), ${ }^{2}$ and $r$ is a squeezing parameter. When the parameter $r$ is greater than $-\left(\frac{1}{2}\right) \ln (2\langle\hat{n}\rangle)$, the photon-number uncertainty is smaller than the standard quantum limit, $\left\langle\Delta \hat{n}^{2}\right\rangle_{\text {SQL }}$ $=\langle\hat{n}\rangle$, while the minimum uncertainty relation, $\left\langle\Delta \hat{n}^{2}\right\rangle\left\langle\Delta \hat{S}^{2}\right\rangle=\left(\frac{1}{4}\right)\langle\hat{C}\rangle^{2}$, is still preserved, where $\widehat{C}=(\hat{n}+1)^{-1 / 2} \hat{a} / 2+$ H.c. is the cosine operator. As $r$ increases, NUS's approach number states which feature the maximum channel capacity in optical communication ${ }^{3}$ and also improve the performance of a gravity-wavedetection interferometer. ${ }^{4}$ The generation of NUS's as well as squeezed states is of potential importance in information transmission and precision-measurement systems.

We have proposed three schemes for generating NUS's: That utilizing self-phase modulation in a Kerr medium, ${ }^{5}$ that using a highly saturated laser oscillator with suppressed pump noise, ${ }^{6}$ and that using negative feedback of the photon number measured by a quantum nondemolition (QND) scheme ${ }^{7}$ to a laser pumping. ${ }^{8}$

In a previous paper on the QND measurement of the photon number ${ }^{7}$ Haus and two of the present authors (N.I. and Y.Y.) demonstrated that the measurement error $\Delta n$ of the signal photon number and the back-action noise $\Delta \phi$ imposed on the signal phase satisfy the minimum uncertainty relation. The result suggests that the signal quantum state after the QND measurement is a NUS with the photon-number uncertainty determined by measurement error $\Delta n$ and the sine uncertainty determined by back-action noise $\Delta \phi$. This paper ${ }^{9}$ examines this prediction within the present framework of quantum theory of measurement, ${ }^{10}$ and discusses the conditions for generating NUS's and number states by state reduction in QND measurement.

The QND measurement scheme is depicted in Fig. 1. The first stage of the QND measurement, i.e., the establishment of a quantum-mechanical correlation between the signal and probe waves, is achieved by a unitary interphase modulation in a Kerr medium. The second stage, i.e., the nonunitary reduction of the state, is realized by homodyne detection of a quadrature-phase amplitude of the probe wave.

The signal (suffix $a$ ) and probe $(b)$ waves are assumed to be initially in coherent states $\hat{\rho}_{a 0}=\left|\alpha_{0}\right\rangle_{a a}\left\langle\alpha_{0}\right|$ and $\hat{\rho}_{b 0}=\left|\beta_{0}\right\rangle_{b b}\left\langle\beta_{0}\right|$, respectively. The initial density operator (DO) of the total system is

$$
\hat{\rho}_{0}=\hat{\rho}_{a 0} \hat{\rho}_{b 0} \text {. }
$$

The interaction Hamiltonian for interphase modulation (IPM) in the optical Kerr medium is expressed by ${ }^{7}$

$$
\hat{H}_{I}=\hbar \chi \hat{n}_{a} \hat{n}_{b},
$$

where $\hat{n}_{a}=\hat{a}^{\dagger} \hat{a}$ and $\hat{n}_{b}=\hat{b}^{\dagger} \hat{b}$ are the photon-number operators of the signal and probe waves. The creation and annihilation operators for the signal and those for the probe are denoted by $\hat{a}^{\dagger}$ and $\hat{a}$, and $\hat{b}^{\dagger}$ and $\hat{b}$, respectively. The coupling constant $\chi$ is proportional to third-order nonlinear susceptibility $\chi^{(3)}\left(\omega_{s} ; \omega_{s},-\omega_{p}, \omega_{p}\right)$. Self-phase modulation processes are assumed to be negligible due to the resonance which only enhances the IPM process. ${ }^{7}$

The equation of motion for the spatial translation operator $\widehat{U}(z)$ in the interaction picture is readily integrated to give

$$
\widehat{U}(z)=\exp \left(i \chi z \hat{n}_{a} \hat{n}_{b} / v\right),
$$

where $v$ is the speed of light in the Kerr medium. After

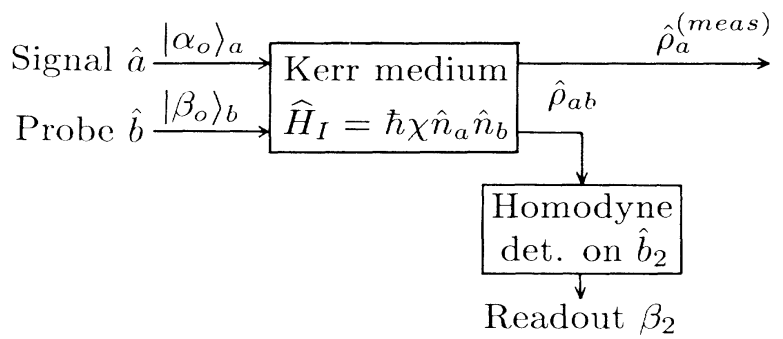

FIG. 1. QND measurement scheme of photon number. 
passage through the Kerr medium of length $L$, the DO for the total system becomes

$$
\hat{\rho}_{a b}=\exp \left(i \mu \hat{n}_{a} \hat{n}_{b}\right) \hat{\rho}_{0} \exp \left(-i \mu \hat{n}_{a} \hat{n}_{b}\right),
$$

where $\mu=\chi L / v$.

The signal and probe photon-number distributions are still Poissonian, $P_{a}(n)=\exp \left(-n_{a 0}\right) n_{a 0}^{n} / n !$ and $P_{b}(n)$ $=\exp \left(-n_{b 0}\right) n_{b 0}^{n} / n !$, where $n_{a 0}=\left|\alpha_{0}\right|^{2}$ and $n_{b 0}=\left|\beta_{0}\right|^{2}$ are the initial mean photon numbers. However, quantum-mechanical correlation has been established between the signal and probe at this stage. The signal experiences an increase in phase uncertainty due to IPM. This can be visualized by a quasiprobability density ( $Q$ representation)

$$
\begin{aligned}
Q_{a}(\alpha) & ={ }_{a}\left\langle\alpha\left|\hat{\rho}_{a}\right| \alpha\right\rangle_{a} \\
& =\sum_{n} P_{b}(n) \exp \left(-\left|\alpha-\alpha_{0} e^{i \mu n}\right|^{2}\right) .
\end{aligned}
$$

Here

$$
\hat{\rho}_{a}=\operatorname{Tr}_{b} \hat{\rho}_{a b}=\sum_{n} P_{b}(n)\left|\alpha_{0} e^{i \mu n}\right\rangle_{a a}\left\langle\alpha_{0} e^{i \mu n}\right|
$$

is the reduced density operator for the signal. The $Q_{a}(\alpha)$ with weak and strong nonlinear interactions are shown in Figs. 2(a) and 2(c).

The sine uncertainty ${ }^{2}$ after IPM calculated from $\hat{\rho}_{a}$ is

$$
\left\langle\Delta \hat{S}_{a}^{2}\right\rangle \approx 1 /\left(4 n_{a 0}\right)+\left[1-\exp \left(-2 \mu^{2} n_{b 0}\right)\right] / 2,
$$

where $n_{a 0} \gg 1$ (say, $n_{a 0}>10$ ) and $\mu \ll 1$ are assumed. Moreover, $\alpha_{0}=\left|\alpha_{0}\right| \exp \left(-i n_{b 0} \sin \mu\right)$ is chosen so that $\left\langle\hat{S}_{a}\right\rangle=0 .{ }^{1}$ The second term is the increase by IPM and is regarded as a back-action noise. ${ }^{7}$ An increase in phase uncertainty is also observed as a decrease in the square of the cosine mean from initial value $\approx 1,{ }^{2}$

$$
\left\langle\widehat{C}_{a}\right\rangle^{2} \approx \exp \left(-\mu^{2} n_{b 0}\right) .
$$

The signal state after the homodyne measurement of a quadrature phase amplitude $\hat{b}_{2}=\left(\hat{b}-\hat{b}^{\dagger}\right) / 2 i$ of the probe is given by the projection into an eigenstate, $\left|\beta_{2}\right\rangle_{b}$, of $\hat{b}_{2}{ }^{10}$ The DO $\hat{\rho}_{a}^{\text {(meas) }}$ of the signal after the readout of $\beta_{2}$ is

$$
\begin{aligned}
\hat{\rho}_{a}^{(\text {meas })}= & N \operatorname{Tr}_{b} \hat{\rho}_{b}^{(\text {read })} \hat{\rho}_{a b} \\
= & N \sum_{k, m}\left\langle k\left|\hat{\rho}_{b}^{(\text {read })}\right| m\right\rangle_{b} \\
& \quad{ }_{b}\left\langle m\left|\hat{\rho}_{b 0}\right| k\right\rangle_{b}\left|\alpha_{0} e^{i \mu m}\right\rangle_{a a}\left\langle\alpha_{0} e^{i \mu k}\right|,
\end{aligned}
$$

where $N$ is a normalization constant determined by $\operatorname{Tr}_{a} \hat{\rho}_{a}^{(\text {meas })}=1 . \quad \hat{\rho}_{b}^{(\mathrm{read})}=\left|\beta_{2}\right\rangle_{b b}\left\langle\beta_{2}\right|$ is the DO corresponding to readout $\beta_{2}$. The DO $\hat{\rho}_{a}^{\text {(meas) }}$ describes the quantum statistical properties of the signal ensemble which features specific readout $\beta_{2}$.

The signal photon-number distribution becomes

$$
P_{a}^{(\text {meas })}(n)=P_{a}(n) G(n),
$$

where

$$
\begin{aligned}
G(n) & =\left.\left.N\right|_{b}\left\langle\beta_{2} \mid \beta_{0} e^{i \mu n}\right\rangle_{b}\right|^{2} \\
& =N^{\prime} \exp \left\{-2\left[\left|\beta_{0}\right| \sin \left(\mu n-n_{a 0} \sin \mu\right)-\beta_{2}\right]^{2}\right\},
\end{aligned}
$$

is a modification factor and $N^{\prime}$ is a constant. Here, $\beta_{0}=\left|\beta_{0}\right| \exp \left(-i n_{a 0} \sin \mu\right)$ is chosen so that the reduced density operator $\hat{\rho}_{b}=\operatorname{Tr}_{a} \hat{\rho}_{a b}$ gives $\left\langle\hat{S}_{b}\right\rangle=0$. This sets the most probable readout for $\hat{b}_{2}$ around $\beta_{2}=0$. In practice,

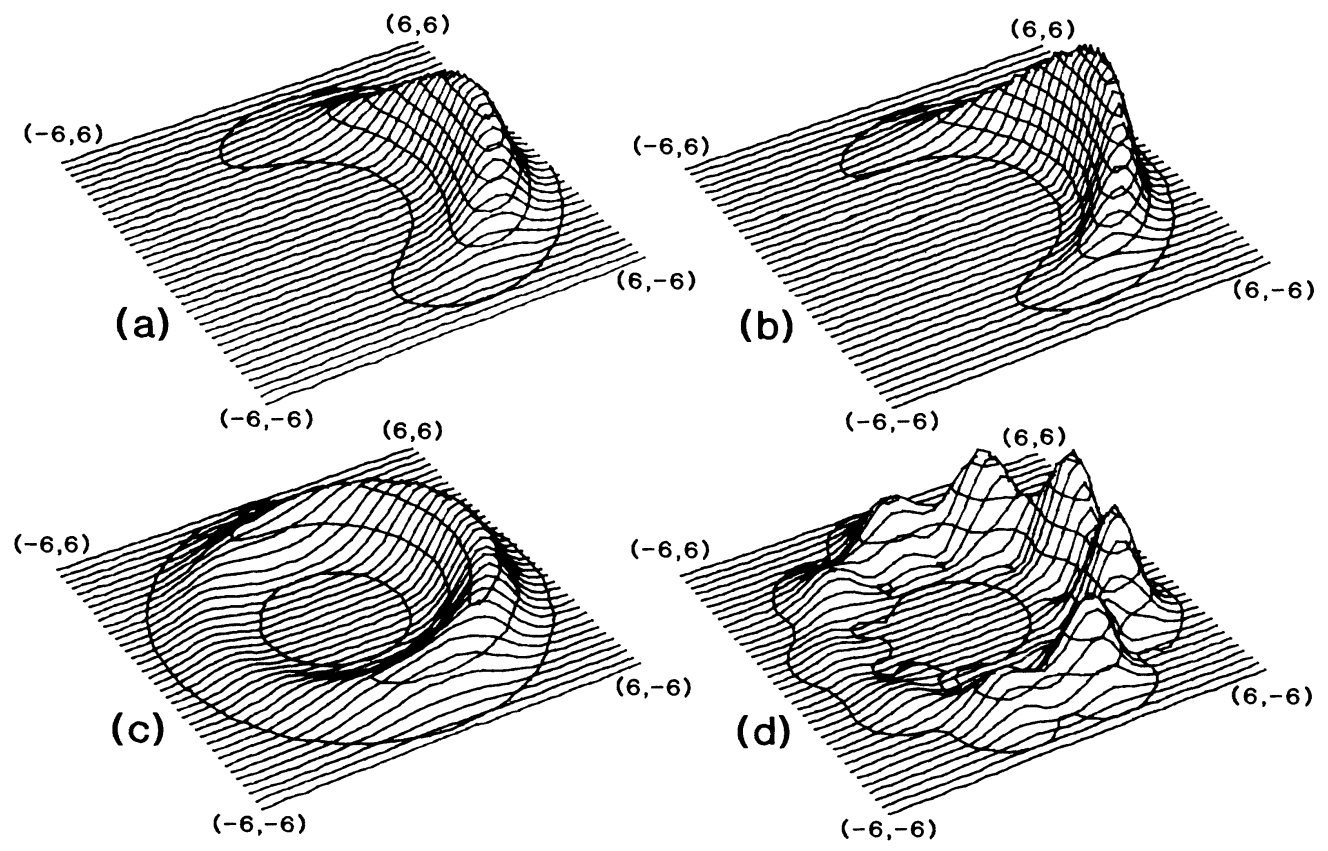

FIG. 2. $Q$ representations of signal states. (a) $Q(\alpha)=\left\langle\alpha\left|\hat{\rho}_{a}\right| \alpha\right\rangle$ before readout, and (b) $Q^{\text {(meas) }}(\alpha)=\left\langle\alpha\left|\hat{\rho}_{a}^{\text {(meas) }}\right| \alpha\right\rangle$ after readout $\beta_{2}=0$, both with weak nonlinear interaction $\mu=0.15$ and $n_{a 0}=n_{b 0}=16$. (c) and (d) are the same as (a) and (b), but both with strong nonlinear interaction $\mu=0.35$. Contours are, from lowest, at $0.005,0.05,0.1,0.15, \ldots, 1.0$. 
this is done by choosing the appropriate local oscillator phase in the homodyne measurement of $\hat{b}_{2}$. The photonnumber distributions for $\hat{\rho}_{a}^{\text {(meas) }}$ are compared with those for $\hat{\rho}_{a}$ in Fig. 3. The photon-number distribution is concentrated more on the average value after the selection based on the readout $\beta_{2}$ when $\mu$ is small, as shown in Fig. 3(a). On the other hand, the distribution with large $\mu$ apparently possesses side lobes as shown in Fig. 3(b). ${ }^{11}$ This is because the factor $G(n)$ is a multiple-peaked function of signal photon-number $n$ with average peak interval $\pi / \mu$. To avoid side lobes in $P_{a}^{(\text {meas })}(n)$, this interval should be far greater than the initial photon-number uncertainty $n_{a 0}^{1 / 2}$. This implies a limit on the nonlinear coupling strength,

$$
\mu<<\pi /\left|\alpha_{0}\right| \text {. }
$$

Moreover, $\left|\beta_{2}\right|$ should be much less than $\left|\beta_{0}\right|$, otherwise the intervals between the nearest-neighbor peaks become so small that a side lobe is unavoidable. However, the probability of obtaining such a large $\left|\beta_{2}\right|$ is negligible as long as $\left|\beta_{0}\right| \gg 1$ since (11) also assures that $\beta_{2}$ is concentrated in the $\left|\beta_{2}\right| \ll\left|\beta_{0}\right|$ region.

For a weak nonlinear interaction $\mu \ll 1, G(n)$ is well approximated around $n=n_{a 0} \operatorname{sinc} \mu \approx n_{a 0}$ by Gaussian function

$$
G(n) \approx N^{\prime} \exp \left\{-2\left[\left|\beta_{0}\right| \mu\left(n-n_{a 0}\right)-\beta_{2}\right]^{2}\right\} .
$$
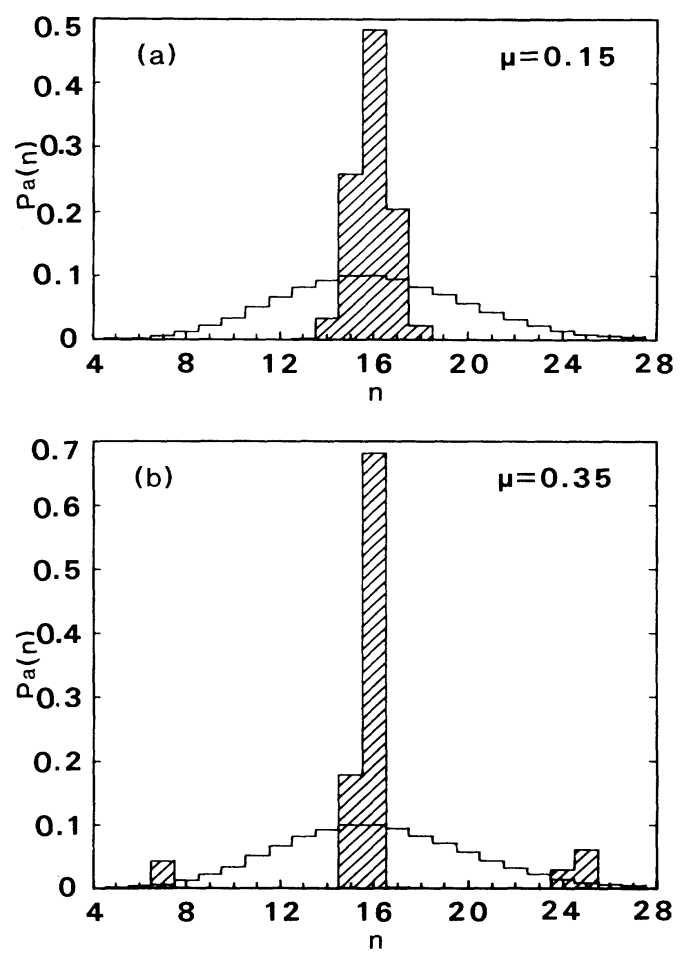

FIG. 3. Photon-number distributions of signal states. (a) Weak nonlinear interaction $\mu=0.15$, and (b) strong nonlinear interaction $\mu=0.35 . \quad n_{a 0}=n_{b 0}=16$. Open distributions are $P_{a}(n)$ before readout, and cross-hatched distributions are $P_{a}^{(\text {meas }}(n)$ after readout $\beta_{2}=0$.
The initial Poisson distribution $P_{a}(n)$ can also be approximated by a Gaussian distribution with variance $n_{a 0}$ when $n_{a 0} \gg 1$. Then $P_{a}^{(\text {meas })}(n)$ is rewritten as

$$
P_{a}^{(\text {meas })}(n) \approx N^{\prime \prime} \exp \left[-\left(n-n_{a 0}-d n\right)^{2} / 2 \sigma^{2}\right],
$$

where $d n=4 \sigma^{2} \mu\left|\beta_{0}\right| \beta_{2} \approx \beta_{2} / \mu\left|\beta_{0}\right|$ is a shift of the mean from $n_{a 0}$ and $N^{\prime \prime}$ is a normalization constant. The photon-number uncertainty is

$$
\left\langle\Delta \hat{n}_{a}^{2}\right\rangle=\sigma^{2}=\left[\left(1 / n_{a 0}\right)+4 \mu^{2} n_{b 0}\right]^{-1} .
$$

This is determined by measurement error $1 / 4 \mu^{2} n_{b 0},{ }^{7}$ and can be arbitrarily reduced by increasing the probe amplitude $\left|\beta_{0}\right|$. The Fano factor $F=\left\langle\Delta \hat{n}_{a}^{2}\right\rangle /\left\langle\hat{n}_{a}\right\rangle$ calculated by (13b) is shown for the case of $\beta_{2}=0$ in Fig. 4 .

The means and variances of the photon number $\hat{n}_{a}$, and the sine $\widehat{S}_{a}$, and cosine $\widehat{C}_{a}$ operators are numerically calculated using $\hat{\rho}_{a}^{\text {(meas) }}$. The Fano factor $F$, the sine uncertainty $\left\langle\Delta \widehat{S}_{a}^{2}\right\rangle$, the square of the cosine mean $\left\langle\widehat{C}_{a}\right\rangle^{2}$, and the number-phase uncertainty product defined by ${ }^{2}$

$$
P_{n S}=\left\langle\Delta \hat{n}_{a}^{2}\right\rangle\left\langle\Delta \hat{S}_{a}^{2}\right\rangle /\left\langle\widehat{C}_{a}\right\rangle^{2},
$$

are calculated from these results and are shown in Fig. 4 by bold curves. Here $\alpha_{0}$ is chosen so that $\left\langle\hat{S}_{a}\right\rangle=0$ is satisfied. ${ }^{1}$ The readout $\beta_{2}=0$ is chosen as an example.

In the region of weak nonlinearity, the Fano factor is reduced by increasing $\mu$. It is then in good agreement with that obtained by (13) assuming that there is no side lobe. In this region, the uncertainty product remains very close to the minimum of 0.25 . $Q_{a}^{\text {(meas) }}(\alpha)$ $={ }_{a}\left\langle\alpha\left|\hat{\rho}_{a}^{(\text {meas })}\right| \alpha\right\rangle_{a}$ shown in Fig. 2(b) exhibits typical characteristics of NUS, namely, squeezing in photon number (radial direction).

The Fano factor increases beyond a certain value for $\mu$. This is attributed to side lobes in the photon-number dis-

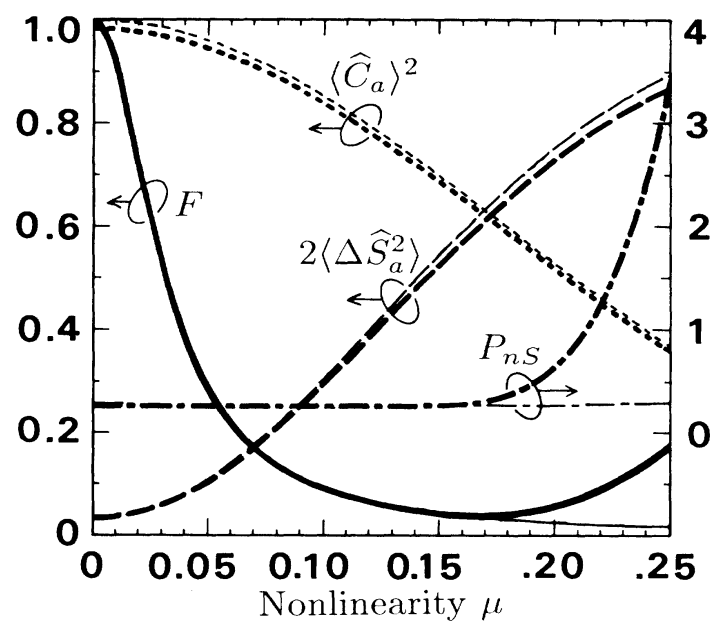

FIG. 4. Fano factor $F$ (solid curves), sine uncertainty (dashed), square of cosine mean (dotted), and number-phase uncertainty product $P_{n S}$ (dashed-dotted) of the state after readout $\beta_{2}=0$. Bold curves are exact numerical results and thin curves are approximate analytical results assuming single-peaked photon-number distribution. $n_{a 0}=n_{b 0}=16$. 
tribution. As a result, the uncertainty product also increases. Spurious substructure is observed in $Q_{a}^{(\text {meas })}(\alpha)$ in Fig. 2(d). These phenomena could be avoided if $\mu$ has been kept small enough to satisfy (11) and $\left|\beta_{0}\right|$ has been increased instead. Phase uncertainty is hardly affected by $\hat{b}_{2}$ measurement on the probe as observed in Fig. 2. In fact, $\left\langle\Delta \hat{S}_{a}^{2}\right\rangle$ and $\left\langle\widehat{C}_{a}\right\rangle^{2}$ of the state after the measurement are well approximated by those before the measurement given in (7) and (8) which are plotted by thin curves in Fig. 4. Therefore, the uncertainty product can be written by using (7), (8), and (13b) as

$$
P_{n S} \approx\left(\frac{1}{4}\right) \sinh \left(\mu^{2} n_{b 0}\right) /\left(\mu^{2} n_{b 0}\right) .
$$

As shown by the thin dashed-dotted curve in Fig. 4, this uncertainty product remains very close to the minimum $\frac{1}{4}$, even when $\mu^{2} n_{b 0}$ is increased to $\frac{1}{4}$ to achieve $\left\langle\Delta \hat{n}_{a}^{2}\right\rangle<1$ in (13b). This is an NUS and also a nearnumber state. A further increase in $\mu^{2} n_{b 0}$ may degrade $P_{n S}$, but still reduces $\left\langle\Delta \hat{n}_{a}^{2}\right\rangle$. Such an increase allows the states to approach number states.
Each signal state after the QND measurement is reduced to a different NUS corresponding to different readout $\beta_{2}$. The probability of finding out the prescribed $\beta_{2}$, i.e., the desirable NUS, decreases with the Fano factor. The continuous generation of the desirable NUS would only be possible when the deviation in $\beta_{2}$ from the prescribed value is fed back to initial state $\hat{\rho}_{a 0},{ }^{12}$ or fed forward to the state after the measurement, $\hat{\rho}_{a}^{\text {(meas) }}$.

In conclusion, the states after QND measurement of the photon number are NUS when the signal and probe are initially in coherent states, and the nonlinear interaction $\mu$ is small enough to eliminate side lobes in the photonnumber distribution. Near-number states, $\left\langle\Delta \hat{n}_{a}^{2}\right\rangle<1$, can be achieved within the NUS, and the states still approach the number states by increasing the probe amplitude $\left|\beta_{0}\right|$.

The authors wish to thank Professor H. P. Yuen of Northwestern University for his careful reading of the manuscript.
${ }^{1}$ R. Jackiw, J. Math. Phys. 9, 339 (1968).

${ }^{2}$ P. Carruthers and M. M. Nieto, Rev. Mod. Phys. 40, 411 (1968).

${ }^{3}$ Y. Yamamoto and H. A. Haus, Rev. Mod. Phys. 58, 1001 (1986).

${ }^{4}$ H. P. Yuen, Phys. Rev. Lett. 56, 2176 (1986).

${ }^{5}$ M. Kitagawa and Y. Yamamoto, Phys. Rev. A 34, 3974 (1986).

${ }^{6}$ Y. Yamamoto, S. Machida, and O. Nilsson, Phys. Rev. A 34, 4025 (1986).

${ }^{7}$ N. Imoto, H. A. Haus, and Y. Yamamoto, Phys. Rev. A 32, 2287 (1985).
${ }^{8}$ Y. Yamamoto, N. Imoto, and S. Machida, Phys. Rev. A 33, 3243 (1986).

${ }^{9}$ Part of this paper was orally presented by Y. Yamamoto, N. Imoto, and S. Machida, Second International Symposium on Foundation of Quantum Mechanics, Tokyo, 1986 (unpublished).

10J. von Neumann, Mathematical Foundations of Quantum Mechanics (Princeton University, Princeton, NJ, 1955).

${ }^{11}$ G. J. Milburn and D. F. Walls, Phys. Rev. A 28, 2065 (1983).

${ }^{12}$ H. A. Haus and Y. Yamamoto, Phys. Rev. A 34, 270 (1986). 